\title{
Politique
}

\section{Tel État, telle fiscalité}

\section{Pierre P. Tremblay}

Numéro 17, hiver 1990

Les nouveaux enjeux du politique

URI : https://id.erudit.org/iderudit/040647ar

DOI : https://doi.org/10.7202/040647ar

Aller au sommaire du numéro

Éditeur(s)

Société québécoise de science politique

ISSN

0711-608X (imprimé)

1918-6584 (numérique)

Découvrir la revue

Citer cet article

Tremblay, P. P. (1990). Tel État, telle fiscalité. Politique, (17), 31-58.

https://doi.org/10.7202/040647ar

Ce document est protégé par la loi sur le droit d'auteur. L'utilisation des services d'Érudit (y compris la reproduction) est assujettie à sa politique d'utilisation que vous pouvez consulter en ligne.

https://apropos.erudit.org/fr/usagers/politique-dutilisation/
Cet article est diffusé et préservé par Érudit.

Érudit est un consortium interuniversitaire sans but lucratif composé de l’Université de Montréal, l'Université Laval et l'Université du Québec à Montréal. Il a pour mission la promotion et la valorisation de la recherche. https://www.erudit.org/fr/ 


\title{
Tel État, telle fiscalité
}

\author{
Pierre P. Tremblay \\ Université du Québec à Montréal
}

Depuis qu'il existe des systèmes fiscaux, les contribuables en ont demandé la réforme. La réforme fiscale est devenue une sorte de mythe. Tels les alchimistes à la recherche de la pierre philosophale, les partis politiques, les groupes de pression, les chercheurs isolés, les illuminés sont en quête depuis des siècles du système d'impôt idéal: souple, juste, efficace, indolore et qui tout en permettant à l'État de verser davantage à chacun et de fournir une quantité accrue de biens et services gratuits, aboutirait à réduire le montant des impôts. (Jean Rivoli, Vive l'Impôt, 1965, p. 45.)

Depuis quelques années déjà, la réforme de la fiscalité est inscrite au programme des sociétés industrialisées. Principale source de financement des dépenses publiques, l'impôt ne permet plus aujourd'hui aux gouvernements d'équilibrer leurs budgets. Les déficits se succèdent et contribuent à faire exploser une dette nationale qui, au Canada, a été évaluée à $53,4 \%$ de la production intérieure brute (PIB) pour l'exercice budgétaire 1988-1989. En dépit d'un optimisme modéré, le ministre des Finances, Michael Wilson, ne prévoit pas d'ici à 1994-1995, de baisse du ratio dette/PIB sous le seuil de 50\% (Wilson, 1990). 
Pour corriger la situation, le réflexe, acquis avec la pratique de l'État-providence, serait de hausser les contributions obligatoires de manière à diminuer les déficits, à contrôler la part du service de la dette dans les crédits annuels de dépenses et à répondre à la demande incrémentale de biens et services. Mais toute hausse des impôts ne peut se faire que dans une conjoncture économique et sociale propice. Or, actuellement, la croissance de l'économie s'essouffle, et le seuil de tolérance du contribuable est presque atteint. Il faudrait un événement grave pour voir ce seuil reculer (Peacock et Wiseman, 1961, p. 27). Au strict plan budgétaire, il faut donc que les gouvernements trouvent de nouvelles formules fiscales qui permettraient de soutenir la cadence de l'accroissement des dépenses publiques.

Mais les difficultés pécuniaires ne peuvent à elles seules transformer des réformes fiscales en enjeux politiques. Or le projet de réforme actuel en est un de taille puisqu'il remet en question la nature et le rôle de l'État actuel et qu'il oblige à revoir la définition d'équité. Dans un de ses récents ouvrages, Alain Minc (1987, p. 210) affirmait que l'impôt est le meilleur aveu qu'une société puisse se faire à elle-même. L'auteur pensait moins aux aspects techniques de la fiscalité, c'est-à-dire le tarif, le taux de prélèvement, voire la structure fiscale, qu'à l'importance de la distribution du fardeau fiscal réel. En effet, la contribution effective de chaque contribuable peut s'avérer contraire à la volonté sociale. Bernard Brachet écrivait à ce sujet: « (...) quel que soit le contenu de la volonté politique, celle-ci se heurte constamment à l'exigence fondamentale du rendement de l'impôt: un prélèvement doctrinalement souhaitable se révèle souvent soit techniquement médiocre soit d'un produit très limité» (1984, p. 634). Ainsi, l'impôt progressif sur le revenu, théoriquement destiné à mieux répartir les revenus et la richesse entre les individus, peut se révéler inefficace en raison de la définition que l'on donne au revenu. Une définition trop restrictive ou trop ambiguë permet à de riches contribuables de ne pas payer d'impôt ou d'en payer très peu. Il faut donc modifier la définition pour augmenter la contribution des plus nantis et générer par le fait même plus de revenu. En attaquant des privilèges, la réforme fiscale devient un enjeu politique. Une fois que 
l'on a constaté le déséquilibre financier, il convient de débusquer les motifs politiques du projet de réforme. C'est la première question préliminaire à laquelle il faut répondre.

Par ailleurs, la démocratie nous a habitués à associer impôt et équité. Qu'en est-il de l'équité? S'agit-il de la répartition du fardeau fiscal selon la capacité fiscale (équité verticale) et les contraintes particulières des individus (équité horizontale)? S'agit-il plutôt du prélèvement des impôts selon le principe de contribution-rétribution: le contribuable est récompensé dans la mesure où il participe à la création de la richesse collective; il est pénalisé en fonction de la consommation qu'il fait (destruction) de cette richesse accumulée? La réponse ne saute pas aux yeux. Il y a donc lieu de s'y arrêter et d'y réfléchir; c'est là la deuxième question préliminaire.

À chaque État sa fiscalité, pourrait-on dire. Une société, dont la philosophie politique dominante est empreinte de valeurs conservatrices et pour qui il est juste de ne pas modifier la distribution de la richesse, opte plus spontanément pour l'impôt à la consommation. En revanche, une société désireuse d'atténuer les écarts entre les individus favorise des prélèvements par impôt progressif sur le revenu (Laufenberger, 1959, p. 133; Matouk, 1987, p. 126). C'est là aussi la thèse que soutient Gabriel Ardant (1965, pp. 87-88) mais dont Pierre Rosanvallon a tracé les limites (1981, p. 40). Pour Rosanvallon, l'impôt de redistribution peut atteindre d'autres résultats que ceux qui étaient escomptés. Il pense que la pratique fiscale inspirée par l'État-providence a donné une société segmentée et balkanisée, d'où ne peut émerger qu'un État clientélaire se substituant peu à peu au premier. Cette affirmation va au-delà de l'équation politique fiscale - philosophie politique; elle met aussi en rapport la nature de l'État et l'utilisation des impôts. En effet, les propos de Rosanvallon laissent entendre que la finalité de l'État commande le recours à des impôts spécifiques. Selon cette thèse, un État dont le rôle est de conserver les richesses et l'environnement pourrait utiliser les impôts à la consommation pour freiner la destruction de ces acquis. D'où la troisième question préliminaire: les politiques fiscales peuvent- 
elles réaliser la finalité de l'État, dans la mesure où celle-ci est explicite et objective?

\section{Pourquoi réformer l'impôt?}

Les motifs qu'invoquent les promoteurs d'une réforme de la fiscalité ne sont pas toujours clairs ni fondés. Souvent aussi, les réformistes tournent ces raisons à leur avantage. Le ministre des Finances du Canada, Michael H. Wilson, déclarait, par exemple, lors du dépôt de son Livre blanc: «Des raisons impérieuses militent en faveur d'une réforme de la fiscalité au Canada. Celle-ci est injuste à maints égards. Elle réduit la capacité de croissance et de création d'emplois du Canada. Elle devient une source de recettes de moins en moins fiable» (juin 1987, p. 1). On aura compris que le ministre, ayant constaté la vacuité des coffres du gouvernement, songe à élargir la matière imposable de manière à minimiser les inconvénients d'une hausse de revenus et à éviter l'odieux de l'abolition de programmes de biens et services publics. En fait, il souhaiterait revenir au statu quo ante au moment où, la croissance aidant, les revenus étaient supérieurs aux dépenses.

\section{Objectifs classiques}

Les objectifs que prétend poursuivre le ministre Wilson dans sa réforme, soit l'équité, la simplicité, la compétitivité, la cohérence, la fiabilité, sont des objectifs classiques (Pechman, 1986, p. 57). Ils permettent au proposant de satisfaire les attentes de tous les payeurs d'impôts, du plus humble au plus riche. L'équité et la simplicité visent le contribuable individuel dont les revenus sont plutôt faibles, alors que la compétitivité, la cohérence et la fiabilité servent davantage à rassurer l'entreprise et les contribuables plus fortunés. Prises individuellement, ces raisons sont vérifiables et peu contestables. Par contre, intégrées dans une structure et une politique fiscales, elles perdent de leur évidence. 
Les objectifs de la réforme fiscale canadienne relèvent d'une conception de l'impôt selon laquelle l'individu doit contribuer en fonction de ce qu'il est et non de ce qu'il fait. Dans cet esprit, un contribuable dont le revenu est élevé doit débourser plus, quels que soient les efforts que lui ont coûtés sa richesse personnelle. Il se trouve ainsi doublement pénalisé.

Pour faire face à la concurrence mondiale actuelle, faut-il abaisser les taux d'imposition et réduire le nombre de paliers, de façon à attirer le plus d'entreprises possible au Canada? Si telle est la solution, le haut niveau des services offerts par l'État-providence devient alors un sérieux handicap à surmonter. $\mathrm{Si}$, au contraire, concurrence veut dire attitude dynamique, créative et novatrice, alors une politique fiscale articulée et judicieuse pourra réussir à diriger les investissements vers la recherche, le développement et la production de biens et services de haute technologie. Cette même fiscalité maintiendra les capitaux disponibles tout au long de l'accomplissement du cycle. Comme cette demande en capitaux pour la recherche et le développement s'accroît sans cesse, les gouvernements pensent stimuler les entreprises et les individus au moyen d'une taxation incitative.

Quant à la simplification de la fiscalité, elle représente un objectif valable quoique secondaire et subordonné à l'impératif d'efficacité économique. D'ailleurs, le contribuable peut avoir recours à des spécialistes capables d'expliquer les aspects les plus difficiles de l'impôt. Mais si l'efficacité peut s'allier à la simplification de l'impôt, on aurait bien tort de s'en priver!

En revanche, l'objectif de cohérence est essentiel, non seulement parce que le Canada est un pays fédéral, mais aussi parce que les moyens mis en oeuvre pour atteindre les objectifs ne doivent pas, dès le départ, être orientés vers des résultats opposés. Par exemple, il me semblerait risqué de vouloir abolir des programmes d'exemptions ou de crédits fiscaux, et ce faisant obliger ceux qui en bénéficient à consacrer une part plus importante de leurs revenus à l'État. Dans de telles conditions, on ne pourrait escompter les voir travailler plus fort pour hausser leur revenu. En raison du besoin de cohérence, il est essentiel que les gouvernements adoptent de solides mesures d'encouragement fiscal à 
l'égard des particuliers et des entreprises qui désirent participer à l'essor de l'économie. Le manque à gagner de l'État (apparent surtout) serait alors largement compensé par une assiette élargie puisque les revenus à cotiser deviendraient plus nombreux.

Pour ce qui est de la fiabilité, seule une économie saine peut la garantir. Or, le développement économique est le fait des personnes et des entreprises autant que de l'État. Par conséquent, la fiabilité des recettes du gouvernement sera assurée dans la mesure où la fiscalité incitera à un effort collectif en vue du développement économique. Fiabilité et bonne économie sont indissociables. En somme, l'argumentation que nous propose le ministre des Finances du Canada est classique mais non compromettante, et elle laisse toute la place aux enjeux économiques. Pour dégager d'autres enjeux, il faut se tourner vers d'autres horizons.

Autres perspectives

D'autres motifs de réforme fiscale que ceux que l'on vient de passer en revue sont proposés par les spécialistes. Brennan et Buchanan (1980, p. 193) suggèrent, par exemple, un motif de réforme de l'impôt fondé sur le critère de Pareto, qui met l'efficacité économique en relation avec l'amélioration du bien-être de l'ensemble de la collectivité. Luc Weber a étendu la portée des critères en l'associant au test de compensation de Hicks et Kaldor; voici ce qu'il en dit:

Selon la formulation de Kaldor, le test de compensation stipule qu'un état $Y$ de l'allocation est socialement préférable à un état $X$ lorsque les individus qui gagnent à ce changement sont potentiellement en mesure de compenser les perdants et conserver malgré tout un gain. L'aptitude des gagnants à compenser les perdants permet de s'assurer que ces derniers n'auraient pas à souffrir du changement. Leur indifférence ainsi acquise, l'un des deux volets de la norme de Pareto est satisfait: personne ne souffre. Le solde bénéficiaire revenant - le cas échéant - aux gagnants, après 
compensation, assure quant à lui le deuxième volet, à savoir l'amélioration du bien-être total (1988, p. 31).

Le raisonnement de Hicks et de Kaldor n'est pas sans faille, Weber en convient, d'autant plus qu'il soutient qu'aucune forme de compensation n'est totale ou parfaite. Cependant, il me semble important de retenir l'effet de compensation relié au seuil d'équilibre. On sait qu'un déséquilibre se produit lorsqu'une partie de la collectivité considère que la compensation de ses efforts est insuffisante. C'est alors qu'il faut recourir à la réforme fiscale.

Si l'on veut utiliser le test de compensation de Hicks et Kaldor dans une perspective politique, il faut le formuler différemment: la distribution $\mathrm{Y}$ du pouvoir est préférable à la distribution $\mathrm{X}$ lorsque les gagnants peuvent convaincre les perdants qu'ils retirent des bénéfices de la situation. Dans cette optique, les véritables vainqueurs du combat fiscal seront ceux qui, après en avoir déterminé les règles, vont recevoir de la politique fiscale un maximum d'avantages et en subir un minimum d'inconvénients. Autrement dit, ils paieront le moins d'impôts possible tout en recevant un maximum de services gouvernementaux. Tant et aussi longtemps que la collectivité estimera que la fiscalité est profitable à la majorité des contribuables, le seuil de tolérance sera respecté. Par contre, dès que l'on percevra un trop grand déséquilibre entre les contributions respectives au financement de l'État, la politique pourra devenir contestable et les privilèges seront remis en question. Tout cela repose en définitive sur la foi en deux principes: l'équité des contributions et l'équité de l'allocation des ressources publiques. Le pouvoir, pour en revenir à la définition que je donnais au début de cet article, permet à ces détenteurs de convaincre les autres contribuables que l'équité est maintenue. Et, comme la notion d'équité est associée à la répartition du fardeau de l'impôt, il s'ensuit que l'enjeu politique de la réforme fiscale, c'est le pouvoir de définir l'équité.

Au Canada, la situation des finances publiques du gouvernement fédéral a atteint un point critique, si bien que pour rééquilibrer tant soit peu les revenus et les dépenses tout en maintenant les programmes actuels, le gouvernement doit augmenter la contri- 
bution des plus riches. Or, ce sont précisément ceux-là qui bénéficient le plus des privilèges fiscaux. Parce qu'ils ont des revenus annuels importants, ces citoyens peuvent paradoxalement les diminuer en toute légalité grâce, entre autres, aux régimes enregistrés d'épargne retraite (REER), aux actions accréditives et aux crédits pour investissement immobilier. Dans le Livre blanc de juin 1987, le ministre des Finances reconnaissait que ces concessions fiscales particulières ont mis à dure épreuve l'équité, l'efficience et la stabilité du produit de l'impôt direct (p. 8). De plus, de l'aveu du ministre, le revenu imposable ne représente aujourd'hui que $60 \%$ du revenu cotisé. La dépense fiscale est de ce fait très élevée: pour la seule année fiscale 1984, ce manque à gagner s'est élevé à 10 milliards de dollars.

Les statistiques fiscales apparaissant au tableau 1, celui-là même que Michael Wilson a utilisé pour illustrer son analyse (1987, p. 9), mettent en évidence un fait significatif. En effet, plus de la moitié des contribuables (52\%) dont le revenu moyen se situe entre 75000 et 100000 dollars est frappée d'un taux moyen d'imposition oscillant entre 20 et $25 \%$. On observe aussi que $21 \%$ de ces contribuables sont imposés à un taux maximal de $15 \%$. En d'autres mots, ils jouissent d'un allégement fiscal considérable puisque, comme en témoigne le tableau 2, leur taux devrait normalement être supérieur à 30\%. À leur décharge, il faut reconnaître que ces contribuables supportent plus de $60 \%$ du fardeau fiscal effectif (tableau 3). Conclusion plutôt paradoxale: ceux qui bénéficient des plus grands privilèges fiscaux sont les plus imposés par le gouvernement fédéral. Alors demander à l'État de diminuer leurs privilèges, c'est aussi lui demander d'accentuer la lourdeur de leur fardeau fiscal. 
Tableau 1: Répartition des contribuables en fonction du revenu et du taux moyen d'impôt fédéral sur leurs revenus, 1984

\begin{tabular}{|c|c|c|c|c|c|c|c|c|c|c|c|}
\hline \multirow[b]{2}{*}{$\begin{array}{l}\text { Revenu } \\
\text { total (1) }\end{array}$} & \multirow[b]{2}{*}{$\begin{array}{c}\text { Nombre } \\
\text { de contribuables }\end{array}$} & \multirow[b]{2}{*}{$\begin{array}{l}\text { Pourcentage } \\
\text { des déclarants }\end{array}$} & \multicolumn{9}{|c|}{ Répartition en pourcentage des particuliers en fonction du taux moyen d'imposition(2) } \\
\hline & & & $\begin{array}{c}\text { Aucun } \\
\text { impôt(3) }\end{array}$ & $\begin{array}{c}\text { Taux } \\
\text { moyen } \\
0-5\end{array}$ & $\begin{array}{c}\text { Taux } \\
\text { moyen } \\
5-10\end{array}$ & $\begin{array}{c}\text { Taux } \\
\text { moyen } \\
10-15\end{array}$ & $\begin{array}{c}\text { Taux } \\
\text { moyen } \\
15-20\end{array}$ & $\begin{array}{c}\text { Taux } \\
\text { moyen } \\
20-25\end{array}$ & $\begin{array}{c}\text { Taux } \\
\text { moyen } \\
25-30\end{array}$ & $\begin{array}{c}\text { Taux } \\
\text { moyen } \\
30+\end{array}$ & Total \\
\hline (Milliers \$) & (milliers) & & \multicolumn{9}{|c|}{ (pour cent) } \\
\hline $0-10$ & 5813 & 37.4 & 73 & 18 & 9 & - & - & - & - & - & 100 \\
\hline $10-20$ & 4178 & 26.9 & 5 & 16 & 53 & 26 & - & - & - & - & 100 \\
\hline $20-30$ & 2752 & 17.7 & 1 & 3 & 25 & 69 & 2 & - & - & - & 100 \\
\hline $30-40$ & 1584 & 10.2 & 1 & 2 & 7 & 66 & 24 & - & - & - & 100 \\
\hline $40-50$ & 661 & 4.3 & 1 & 2 & 5 & 40 & 51 & 1 & - & - & 100 \\
\hline $50-75$ & 396 & 2.5 & 2 & 2 & 5 & 15 & 64 & 12 & - & - & 100 \\
\hline $75-100$ & 83 & 0.5 & 2 & 3 & 5 & 11 & 26 & 52 & 1 & - & 100 \\
\hline $100-150$ & 48 & 0.3 & 4 & 4 & 5 & 10 & 16 & 40 & 22 & - & 100 \\
\hline 150 et plus & 33 & 0.2 & 4 & 5 & 5 & 9 & 16 & 19 & 34 & 8 & 100 \\
\hline Total & 15552 & 100.0 & 29 & 12 & 23 & 28 & 7 & 1 & - & - & 100 \\
\hline
\end{tabular}

Source: Michael Wilson, La réforme de l'impót direct, Ottawa, 1987, p. 10.

(1) Revenu total = revenu de toutes provenances. (2) Taux moyen d'impôt à payer avant crédit d'impôts pour enfants. (3) Contribuables ayant moins de $\$ 10$ ou $0.1 \%$ du revenu à payer en impôt fédéral. 
Tableau 2: Distribution du taux d'imposition au Canada, 1961-1987 Années choisies (décile et pourcentage)

\begin{tabular}{lcccccccccc}
\hline Année & 1 & 2 & 3 & 4 & 5 & 6 & 7 & 8 & 9 & 10 \\
\hline 1961 & 19.9 & 19.9 & 19.9 & 20.4 & 20.9 & 21.3 & 23.2 & 23.3 & 24.6 & 32.9 \\
1969 & 20.6 & 21.5 & 25.1 & 26.4 & 27.8 & 27.4 & 27.4 & 29.7 & 30.6 & 38.2 \\
1972 & 17.0 & 18.7 & 23.8 & 26.2 & 28.0 & 29.6 & 29.7 & 31.0 & 32.2 & 38.1 \\
1974 & 21.4 & 21.5 & 26.8 & 27.6 & 29.7 & 30.2 & 30.9 & 32.5 & 33.9 & 39.6 \\
1976 & 15.7 & 20.9 & 25.2 & 27.4 & 27.0 & 27.3 & 27.9 & 31.0 & 33.4 & 38.8 \\
1978 & 16.3 & 22.7 & 26.4 & 29.1 & 29.9 & 30.0 & 30.7 & 31.7 & 32.8 & 33.2 \\
1981 & 13.6 & 19.5 & 24.4 & 28.1 & 29.9 & 31.4 & 32.8 & 33.7 & 34.7 & 38.2 \\
1984 & 12.7 & 17.3 & 21.3 & 26.6 & 29.4 & 30.9 & 31.9 & 32.5 & 34.9 & 36.2 \\
1987 & 16.7 & 19.9 & 24.5 & 28.4 & 30.9 & 32.1 & 32.9 & 34.0 & 35.6 & 35.6 \\
& & & & & & & & & & \\
\hline
\end{tabular}

Source: Fraser Institute Canadian Tax Simulator (CANTASIM) 
Tableau 3: Distribution du fardeau fiscal au Canada, 1961-1987 Années choisies (décile et pourcentage)

\begin{tabular}{lcccccccccc}
\hline Année & 1 & 2 & 3 & 4 & 5 & 6 & 7 & 8 & 9 & 10 \\
\hline 1961 & 2.1 & 2.1 & 4.5 & 5.7 & 6.7 & 7.7 & 10.5 & 10.7 & 14.6 & 35.6 \\
1969 & 1.1 & 2.0 & 4.0 & 5.3 & 6.9 & 7.7 & 9.4 & 11.8 & 15.0 & 36.9 \\
1972 & 0.6 & 1.9 & 3.5 & 5.1 & 6.7 & 8.4 & 9.8 & 12.4 & 16.1 & 35.5 \\
1974 & 0.6 & 1.8 & 3.7 & 5.1 & 6.7 & 8.4 & 9.6 & 13.0 & 15.9 & 35.5 \\
1976 & 0.6 & 1.9 & 3.6 & 5.1 & 6.0 & 7.6 & 8.6 & 12.9 & 17.3 & 36.3 \\
1978 & 0.7 & 2.2 & 4.2 & 6.2 & 7.7 & 8.8 & 10.8 & 13.0 & 16.6 & 30.0 \\
1981 & 0.6 & 1.9 & 3.8 & 5.5 & 7.1 & 8.9 & 11.0 & 13.4 & 16.8 & 31.1 \\
1984 & 0.6 & 1.6 & 3.0 & 5.1 & 7.2 & 8.9 & 10.9 & 13.2 & 21.0 & 28.5 \\
1987 & 0.7 & 1.9 & 3.3 & 5.2 & 7.3 & 9.1 & 11.2 & 13.9 & 23.7 & 23.7 \\
\hline
\end{tabular}

Source: Fraser Institute Canadian Tax Simulator (CANTASIM) 
Le dilemme devant lequel se trouve le gouvernement ne se limite pas à l'équation ci-dessus. Les abris fiscaux constituent aussi des investissements dont l'effet de stimulation économique n'est pas négligeable. L'État canadien est donc en présence d'un conflit entre l'équité et l'efficacité (Weber, 1988, p. 112). Comme solution, le ministre Wilson a choisi une réforme en deux temps: diminution d'abord des taux de prélèvement et abolition de certains privilèges; introduction ensuite d'une nouvelle taxe de vente, la taxe sur les produits et services (TPS). Belle astuce. En se retranchant derrière la neutralité de la réforme, le gouvernement fédéral modifie la structure fiscale: il transfère une partie du poids de l'impôt direct sur le revenu dans l'impôt indirect à la consommation. Du point de vue économique, cette manoeuvre se justifie puisque la combinaison des trois facteurs: taux de chômage réel élevé, vieillissement de la population et ralentissement du taux de croissance, entraînera une diminution du nombre et de l'importance des revenus imposables. Autrement dit, le processus de rétrécissement de la matière imposable est déjà enclenché, et il faut l'arrêter. À cet égard, le champ de la consommation présente de belles possibilités, car il contient beaucoup plus d'objets imposables. La stabilité et l'efficacité des prélèvements s'améliorent d'autant. Cependant, le caractère particulier des impôts à la consommation, c'est-à-dire la possibilité de les transférer, pose un problème de taille sur le plan des objectifs d'équité.

Selon la théorie fiscale, l'impôt sur la vente ou sur l'achat étant une contribution indirecte (Mehl et Beltrame, p. 185), il est supporté et payé indirectement par le consommateur. Le fardeau fiscal appliqué à la consommation est donc susceptible d'être répercuté du contribuable légal au contribuable effectif. Voilà une première caractéristique. En outre, cet impôt est proportionnel, donc éventuellement régressif, c'est-à-dire que, pour un même achat, le prélèvement pèse plus lourdement sur les pauvres que sur les riches. Si les contribuables les plus riches (dont la capacité contributive est la plus élevée) constituent la majorité de ceux qui peuvent transférer leurs impôts, ce seront eux finalement les véritables gagnants de la réforme. D'une part, leurs impôts sur le revenu seront abaissés et d'autre part, leurs impôts à la consomma- 
tion pourront être répercutés. Même dans l'hypothèse d'une incapacité de les répercuter, ces impôts demeureront plus légers à supporter en raison du caractère régressif des impôts à la consommation.

Si on suppose que la réforme de l'impôt est basée sur un enjeu politique plutôt que sur des motifs économiques, on risque d'y voir une stratégie visant à maintenir la distribution du fardeau fiscal réel dans un statu quo. Il ne faudrait jamais oublier que les promoteurs du projet de réforme ont une conception libérale de l'économie.

\section{Une question d'équité}

Les notions fiscales d'universalité, de proportionnalité et de progressivité sont relativement claires (Dafflon et Weber, 1984, p. 727). Il n'en est pas ainsi du concept d'équité, qui se définit, on l'a vu, verticalement ou horizontalement. À la verticale, chaque contribuable supporte un fardeau fiscal égal à sa capacité réelle. Prémisse de l'impôt progressif sur le revenu, que justifient la nécessité d'égaliser le sacrifice de l'impôt et le besoin de solidarité sociale (Salin, 1985, p. 37). Par contre, à l'horizontale, les contribuables qui jouissent d'un avoir équivalent sont censés subir un prélèvement égal. L'équité verticale est d'application difficile en raison même de la technique fiscale. Quant à l'équité horizontale, elle oblige le législateur à disposer d'une batterie de pondérations qui tiennent compte des obligations et contraintes propres à chaque contribuable. Idéalement, un impôt équitable serait à la fois progressif et personnalisé. Par surcroît, cet impôt devrait égaliser les chances et redistribuer la richesse.

Lors du dépôt de son projet de réforme, le ministre des Finances (juin 1987, p. 3) a attribué la cause des problèmes budgétaires actuels au fait que les Canadiens ne respectent pas le régime fiscal. Ce régime, fruit des politiques fiscales des gouvernements qui se sont succédé depuis au moins deux décennies, permet à beaucoup de sociétés rentables d'éviter de payer toute leur part de l'impôt. Il en est de même pour les individus qui, en raison d'un 
revenu suffisamment élevé, profitent des abris fiscaux. Toutes ces dépenses fiscales, l'État doit les financer soit en empruntant, soit en transférant une partie de cet impôt à d'autres contribuables. Résultat: malgré un taux marginal d'imposition élevé, nombre de contribuables peuvent diminuer leurs revenus imposables et, par conséquent, réduire leurs impôts. À partir de ces observations sur la structure fiscale du gouvernement américain, Joseph A. Pechman (1986, pp. 1-2) déduit que l'impôt aux États-Unis est en réalité relativement progressif en dépit d'une structure nettement progressive. La cause: la possibilité de réduire la partie imposable des revenus individuels.

\section{Progressivité de l'impôt}

La finalité sociale de l'impôt entretient le mythe de la progressivité de l'impôt sur le revenu. Cependant, tous n'en sont pas dupes et voient même certains avantages à l'abandonner:

Est-il utopique de vouloir supprimer la progressivité de l'impôt? Tel n'est certainement pas l'avis de ceux - nombreux - qui proposent aux États-Unis le remplacement de l'impôt progressif par l'impôt proportionnel. Ainsi en 1983, le sénateur démocrate Denni Deconcivi a déposé un projet, mis au point par l'économiste Robert Hall et le spécialiste de science politique Alvin Rabushke (de l'Institut Hoover), d'après lequel il existerait un taux unique de 19\% (d'ailleurs appliqué également à l'impôt sur les bénéfices des sociétés), avec un abattement à la base de 8500 dollars pour une famille de quatre personnes, mais en supprimant toutes les exemptions actuelles. Un autre projet a été déposé par deux parlementaires, également démocrates, Richard Gephardt et Bil Bradley, proposant de ne laisser subsister que trois taux, 14, 26 et 30\%. Les Républicains Jack Kemp et Robert Kaster ont, pour leur part, fait des propositions similaires. Le secrétaire au Trésor lui-même les a suivis en proposant, en novembre 1984, des taux de 15, 25 et 35\%. Sa proposition a été retenue et annoncée par le président Reagan en mai 1985 (Salin 1985, pp. 65-66). 
En extrapolant à partir des propos ci-dessus, il est raisonnable de penser que l'impôt souhaitable serait un impôt à taux unique, donc proportionnel. Cette tendance se rattache au constat d'échec, auquel en arrivent les auteurs des différents projets. Pour eux, la progressivité ne fonctionne tout simplement pas. Rappelons qu'au Canada le premier volet de la réforme Wilson ramène aussi le nombre de taux applicables à l'impôt sur le revenu des individus à trois: 17,26 et $29 \%$. Cependant l'échec étant socialement et politiquement inavouable, on préfère d'emblée relier la réduction du nombre de paliers d'imposition à un objectif plus évasif, celui de simplifier le système fiscal.

\section{Effet distributif de l'impôt}

Il n'y a pas que la progressivité de l'impôt qui inspire des doutes. Son effet distributif en soulève aussi. Aux États-Unis, par exemple, on a observé que, sur une période de 30 ans et pour les $15 \%$ des contribuables les plus riches, la part du revenu après impôt n'avait pas diminué (Pechman, 1986, p. 25). Ces riches contribuables accaparaient, en 1952, 30\% de l'ensemble des revenus après impôt, part qui, en 1981, a grimpé jusqu'à 35\%. Les mêmes statistiques soutiennent que les plus riches d'entre les riches ne sont nullement affectés par des impôts dits distributifs. Par contre, la redistribution a été surtout profitable à des contribuables légèrement moins fortunés, mais dont les revenus se situent encore bien au-delà de la moyenne américaine. La raison de ce maigre succès des politiques distributives réside dans la nonprogressivité effective de l'impôt sur les revenus au cours de cette même période. À ce sujet, Pechman constate $(1986$, p. 24) que le taux effectif de l'impôt sur le revenu du premier $1 \%$ des contribuables les plus fortunés est passé de $33 \%$ en 1952 à $30 \%$ en 1981, soit une baisse réelle de 3\%. Perte qui a été compensée toutefois par l'augmentation du fardeau fiscal qu'a supportée la catégorie suivante de contribuables, lesquels ont vu leur taux croître de $20 \%$ à $23 \%$. Pechman cite aussi une étude de simulation menée à l'aide de trois hypothèses de progressivité (faible, moyen- 
ne et forte). Cette étude démontre que, selon l'hypothèse la plus forte, la courbe de distribution (courbe de Lorenz et coefficient de Gini) ne se déplace que de $10 \%$ vers la droite de la distribution d'égalité complète. Par contre, selon l'hypothèse de progressivité moyenne, la courbe de Lorenz ne bouge presque pas. Des études antérieures à celle que cite Pechman, entre autres celle de Tait (1967) et Nickson (1971), sont arrivées à des conclusions similaires. Tait (1967, p. 6), qui comparait la distribution de GrandeBretagne à celle des États-Unis, a observé que l'inégalité était beaucoup plus accentuée en Grande-Bretagne, en dépit d'une plus longue tradition sociale-démocrate chez les Britanniques. Il faut toutefois tenir compte de l'histoire: la concentration de la richesse étant plus ancienne chez les Britanniques, elle est forcément beaucoup plus cristallisée.

En ce domaine, la situation du Canada se compare à celle des États-Unis. De 1961 à 1987, comme le suggère le tableau 4, les contribuables les plus fortunés (10e décile) ont vu leur part du revenu total passer de $27,2 \%$ à $21,8 \%$, alors que leur taux moyen de taxation s'élevait de $32,9 \%$ à $35,6 \%$. Par contre, leur part du fardeau réel diminuait, de façon étonnante, de presque 12 points de pourcentage, soit de $35,6 \%$ à $23,7 \%$. On peut en déduire que la perte subie dans la répartition du revenu a été compensée par une diminution du fardeau fiscal dans une proportion de 1 à 2 . Il y aurait donc eu double redistribution, la seconde ayant été beaucoup plus profitable compte tenu d'une définition plutôt restrictive du revenu. Bref, la progressivité se serait atténuée en faveur de ce groupe de contribuables. Par ailleurs, les contribuables moins fortunés mais appartenant quand même aux catégories les plus riches (8e et 9e déciles) ont vu leur part de revenu croître au cours de la même période. L'enrichissement le plus marqué a été le fait du $9 \mathrm{e}$ décile dont la valeur s'est accrue de $14,9 \%$ à $21,8 \%$. En revanche, leur fardeau fiscal s'est lui aussi accru de $14,6 \%$ à $23,7 \%$.

Quant aux Canadiens les plus pauvres, comme le montre le tableau 4, leur situation s'est détériorée par rapport à l'ensemble de la population. De 1961 à 1987, ces contribuables, à l'exception de ceux du second décile, ont vu leur part de richesse diminuer. $\mathrm{Au}$ début de la période, soit en 1961 , ils recevaient entre 2,6 et $5,6 \%$ 
du revenu; en 1987 leur fourchette de distribution n'était plus que de 1,4 à $4,4 \%$. En contrepartie, le fardeau fiscal supporté a lui aussi diminué de 2,1 et $4,5 \%$ à 0,7 et $3,3 \%$. Cette baisse est probablement due au jeu de la progressivité pour une part et pour l'autre part, aux piètres résultats des politiques de redistribution, notamment celles qui concernent les programmes gouvernementaux d'allocation des ressources. La classe moyenne au Canada, si j'interprète bien les tableaux 2,3 et 4 , connaît le sort le moins enviable. La part du revenu global qui lui revient a diminué (déciles 4 et 5) ou, tout au mieux, est demeurée stable (déciles 6 et 7). Par contre, toutes les catégories de cette classe de contribuables ont vu leur taux moyen d'imposition augmenter et leur fardeau réel s'accroître.

Il ressort des observations qui précèdent qu'une progressivité plus accentuée dans les taux moyens de prélèvement et les fardeaux réels n'a pas réussi en 25 ans à mieux équilibrer le partage des revenus des Canadiens. On doit donc tirer deux conclusions. La première est que l'impôt progressif sur le revenu semble avoir peu d'effets sur la distribution des revenus. La seconde est qu'une structure du taux de prélèvement, basée sur l'équité verticale, ne garantit pas une juste distribution du fardeau fiscal effectif. Avant de poursuivre tout projet de réforme fiscale, peut-être faudrait-il d'abord repenser le concept d'équité de façon à mieux redistribuer les ressources et éviter une situation sociale au sujet de laquelle Alan Tait écrivait:

Such inequality in the ownership of wealth that gives to its owners freedom of pattern in consumption and the choice of undertaking unusual 'hump' expenditures on education or health, bestows security and power on its possessors, and influences the pattern of saving and risk-taking, which is one of the mainsprings of economic growth, is the concern of everyone. The future of such inequality in our society is of concern to ourselves and our descendants (Tait, 1987, p. 6). 
Tableau 4: Distribution du revenu au Canada, 1961-1987

Années choisies (décile et pourcentage)

\begin{tabular}{lcccccccccc}
\hline Année & 1 & 2 & 3 & 4 & 5 & 6 & 7 & 8 & 9 & 10 \\
\hline 1961 & 2.6 & 2.6 & 5.6 & 7.0 & 8.1 & 9.1 & 11.3 & 11.5 & 14.9 & 27.2 \\
1969 & 1.6 & 2.9 & 4.9 & 6.2 & 7.7 & 8.7 & 10.3 & 12.3 & 15.2 & 30.0 \\
1972 & 1.0 & 3.3 & 4.7 & 6.2 & 7.5 & 8.9 & 10.4 & 12.7 & 15.8 & 29.4 \\
1974 & 1.0 & 2.8 & 4.6 & 6.2 & 7.5 & 9.2 & 10.3 & 13.3 & 15.6 & 29.8 \\
1976 & 1.2 & 3.0 & 4.6 & 5.9 & 7.1 & 8.9 & 9.8 & 13.2 & 16.5 & 29.8 \\
1978 & 1.3 & 3.0 & 4.9 & 6.6 & 8.0 & 9.1 & 10.9 & 12.7 & 15.7 & 28.0 \\
1981 & 1.4 & 3.2 & 5.1 & 6.5 & 7.8 & 9.3 & 11.0 & 13.1 & 15.9 & 26.8 \\
1984 & 1.4 & 3.0 & 4.5 & 6.1 & 7.8 & 9.1 & 10.9 & 12.9 & 19.2 & 25.1 \\
1987 & 1.4 & 3.1 & 4.4 & 6.0 & 7.7 & 9.2 & 11.2 & 13.4 & 21.8 & 21.8 \\
& & & & & & & & & & \\
\hline
\end{tabular}

Source: Fraser Institute Canadian Tax Simulator (CANTASIM). 
On connaît d'autres inconvénients à l'impôt progressif sur le revenu. Entre autres, il décourage l'effort au travail (Salin, 1985 , p. 43) et incite à l'évasion fiscale. Ces inconvénients amènent les contribuables à voir l'impôt, non plus comme une juste mesure de solidarité sociale, mais comme une pénalité à la production. Dans une conjoncture où le rythme et la croissance de la production nationale s'essoufflent, une question se pose: est-il approprié d'accentuer les prélèvements obligatoires sur le revenu, ou ne serait-il pas plus sage et équitable d'encourager les producteurs et de ralentir la destruction de la richesse (la consommation)? La réponse à cette question est aussi une réponse au problème de la définition de l'équité fiscale.

\section{Tel État, telle fiscalité}

L'État moderne est un État producteur de biens et de services, dont il doit assurer la distribution équitable entre les citoyens. C'est là le modèle étatique le plus achevé, et peu de sociétés industrialisées s'en écartent encore. Cet État protège, produit et décide. En dépit des critiques acerbes qui lui sont adressées, il ne semble pas être question de le démanteler. Tout au plus, souhaite-t-on parfois qu'il soit plus modeste (Crozier, 1987, p. 10). On constate d'ailleurs régulièrement que les demandes de privatisation des services publics ne résistent pas aux soubresauts de l'économie. On peut même dire qu'une économie florissante et dynamique illusionne les entrepreneurs en leur laissant croire qu'ils pourraient faire mieux et plus que ne le fait l'appareil public. Quand survient le ralentissement économique, les adeptes du marché privé remballent vite leurs prétentions, aussi vastes que le profit qu'ils escomptaient. Même dans l'euphorie affairiste, le rôle moteur de l'État n'a jamais été contesté. On lui demandait de se départir de sa force de production de biens et services; en revanche, on l'incitait à demeurer le générateur de la demande. Comme l'a remarqué Crozier (1987), on ne contestait ni la présence ni le rôle des gouvernements mais leur propension à tout diriger et réglementer. En somme, le projet libéral, puisque c'est le modèle dont 
il s'agit, s'oppose à l'étatisme, et non à l'État (Sorman, 1984, p. II).

Si au fond des choses il n'y a pas une volonté déterminée d'abandonner le concept de l'État-providence, il reste que tous les pays industrialisés qui le pratiquent connaissent de sérieuses difficultés budgétaires. L'économie publique requiert un accroissement soutenu des dépenses. Pendant ce temps, les ponctions fiscales exercées sur l'économie privée ont déjà atteint un seuil critique et font décroître le rendement de l'impôt (Laffer, 1981). Le problème de l'État est donc avant tout un problème de financement. La solution est une réforme fiscale dont les enjeux débordent le cadre étroit de la comptabilité et englobent l'économique, le social et le politique.

L'État-providence a pour finalité l'optimisation du capital bonheur des citoyens, irréalisable sans associer le développement économique au développement social. Cette mission providentielle a nécessité un élargissement des fonctions de la fiscalité. $\mathrm{Au}$ paiement du coût des services publics, il a fallu ajouter la croissance de l'économie et la redistribution des richesses produites.

La fonction de régulation de l'économie est plus délicate que la simple fonction de prélèvement de fonds. Elle peut tout aussi bien causer l'appauvrissement que favoriser l'enrichissement. Des impôts trop lourds découragent en effet toutes les formes de productivité et entraînent le développement d'activités économiques souterraines dont tous finissent par payer le prix. En revanche, une imposition judicieusement choisie et répartie de façon équilibrée pourrait stimuler les activités économiques, tout en aidant les secteurs plus faibles sans freiner pour autant les secteurs industriels forts. Ce sont là les défis de toute politique économique et auxquels participe une politique fiscale liée à la philosophie interventionniste des États. Vu sous cet angle, l'impôt devient un levier de la production de la richesse collective. Les économistes en parlent comme de la croissance du produit national brut (PNB) ou du produit intérieur brut (PIB). Cependant, l'efficacité de cet instrument ne fait pas consensus. Certains constatent un lien de cause à effet entre l'augmentation des revenus fiscaux et la croissance des productions nationales. D'autres, au contraire, y voient 
une entrave aux performances du marché libre. Il reste que l'impôt et son corollaire, la dépense fiscale, servent de bailleurs de fonds aux entreprises qui investissent et tirent des profits. En ce sens, l'État est un des banquiers (peut-être le plus sûr et le plus tolérant) de l'entreprise. Cette fonction permet d'ailleurs de douter (Salin, 1985) des prétentions qu'a l'autorité étatique de mieux allouer les ressources que le marché ne saurait le faire. Mais, contrairement à Salin, je ne crois pas que le prélèvement fiscal soit une spoliation contre nature. L'impôt pourrait être une excellente façon de civiliser la «lutte pour la survie» dans la mesure où la technique qui est la sienne renforce le caractère distributif. La régulation économique a l'inconvénient d'agir à sens unique: elle ne donne à l'État que peu de moyens de participer aux profits qu'il contribue à structurer. L'entreprise, petite ou grande, nationale ou multinationale, n'aura aucun scrupule à solliciter et à recevoir des subventions directes ou indirectes de la part des pouvoirs publics. Par la suite, elle fera des prodiges d'ingéniosité pour minimiser et cacher l'importance de ses revenus, à partir desquels l'État pourrait redistribuer une part du profit auquel il a contribué.

On a dit de l'impôt qu'il a aussi pour fonction de mieux redistribuer les ressources et de tendre vers la réalisation de l'égalité des chances dans une collectivité. Cette fonction, les gouvernements l'exercent par divers moyens. Ils interviennent soit par la production de biens et de services, soit par des programmes de paiements de transfert ou encore à l'aide d'une fiscalité progressive. Dans le premier cas, l'État doit décider de l'équilibre à maintenir entre ses programmes de développement économique et ses programmes de développement social. La croissance économique profite surtout à ceux qui détiennent déjà un pouvoir économique puisqu'ils sont les éléments moteurs de ce développement. Le développement social, en particulier en raison de son caractère universel, profite à tous sans distinction. Ce qu'il faut surtout souligner, c'est qu'en mettant l'accent sur le développement économique, les gouvernements accroissent les inégalités dans la distribution de la richesse. Cela tient au fait que les instruments fiscaux de la prospérité économique profiteraient davantage aux plus riches qu'aux autres citoyens. Hubert Brochier croyait que l'inégalité était 
une caractéristique des économies capitalistes (1950). Pour ma part, j'attribuerais plutôt cette caractéristique au développement économique de l'État.

Une redistribution ne peut être efficace que si elle touche les revenus et non la richesse. Bien que les deux concepts soient difficiles à cerner et à définir, le premier est plus aisément repérable grâce à son lien direct avec la consommation ou ce que d'aucuns nomment le «pouvoir d'achat». En effet, la richesse d'un individu et, à plus forte raison celle d'une société, ne peut se mesurer seulement en termes de produit national brut (PNB) (Tait, 1967). L'éducation, l'habileté professionnelle ou personnelle, les aptitudes physiques sont des richesses qui n'apparaissent pas nécessairement au tableau de la comptabilité nationale, bien qu'elles soient réelles et fournissent d'indéniables avantages à ceux qui les possèdent. Tout État, aussi puissant ou aussi coercitif soit-il, ne peut trouver une formule magique de redistribution de cette richesse, puisqu'elle est inhérente à chaque individu. L'État démocratique a donc une capacité limitée de rééquilibrer la distribution de la richesse, étant donné qu'il ne peut intervenir que sur des revenus et richesses tangibles et mesurables avec des instruments d'évaluation qu'il a forgés (Musgrave, 1986, p. 81).

La récession économique des premières années de la décennie de 1980 a remis en cause les programmes de dépenses de l'État actuel et du même coup montré les limites de sa capacité de production. Pendant ce temps, l'environnement a continué son envoi de messages de détresse et, en dépit d'innombrables programmes visant le développement de l'individu, on n'a pas amélioré l'autonomie des personnes. Face à cette réalité socio-économique et politique, l'État et la fiscalité doivent donc se transformer. À la question «pourquoi l'impôt», il faudra bientôt répondre qu'en plus de ses finalités traditionnelles, il doit servir à contrer une consommation désordonnée et abusive. Autrement dit, à sauvegarder l'acquis.

L'État-providence est le fruit de la croyance en une capacité illimitée de production de biens et services et il est indissociable de l'abondance des ressources. Lorsque les ressources se raréfient, on assiste à une nouvelle prise de conscience des problèmes 
écologiques et environnementaux. On le voit actuellement. Ces questions écologiques révèlent une peur consciente des risques d'auto-destruction et sont peut-être un catalyseur capable de réorienter les gouvernements vers de nouveaux rôles. Ce sont de nouvelles données de l'enjeu politique. Crozier (1987, p. 309) écrivait que la complexité des affaires de la collectivité déborde le cadre des seules compétences de l'appareil étatique, et que la meilleure machine demeure celle de l'individu dans ses relations avec les autres et la société. Dans cette perspective, la tâche du politique (État) est de «créer les conditions permettant aux individus de décider pour eux-mêmes entre eux et en aidant à dégager un bien commun perpétuellement remis en question». Telle doit devenir la tâche essentielle du politique. C'est là la fonction et le rôle d'un Étatarbitre. La détermination du rôle de l'État est donc un préalable à la réforme de la fiscalité parce que l'État doit utiliser une fiscalité propre à garantir l'accomplissement de ses finalités.

\section{Conclusion}

La restructuration des paliers d'imposition sur le revenu est insuffisante pour faire une véritable réforme. On l'a vu, la technique fiscale et les programmes de déductions annihilent rapidement les effets d'une restructuration. Par surcroît, un alourdissement du fardeau fiscal pourrait entraîner un ralentissement de l'économie et se solder par une augmentation du déficit et de la dette. En raison de son coût, la dette réduit en retour la marge de manoeuvre de l'État et, par conséquent, sa capacité de répondre à de nouveaux besoins, toujours plus nombreux. Dans cette conjoncture, l'État se doit de trouver une nouvelle façon d'imposer qui lui permette de procéder plus efficacement à l'allocation, à la redistribution, à la stabilisation de l'économie, en même temps qu'à la régulation de la consommation des biens.

L'impasse idéologique dans laquelle se trouvent les gouvernements à l'heure actuelle est le principal obstacle à une refonte de l'impôt. James M. Buchanan (1980, p. 193) écrit à ce propos: 
Once the benevolent despotism model of governmental behavior is abandoned, the orthodox suggestions for tax reform that tend to emerge from the equi-revenu analytical framework cannot stand alone. Alternative tax institutions and rules must be evaluated on criteria other than efficiency and equity, although of course, these standard objectives remain relevant.

Il ressort de ces propos que le modèle de l'État-providence n'est peut-être plus le modèle à perpétuer et que les concepts d'efficacité et d'équité sur lesquels reposent les politiques ont besoin d'être revus et réinterprétés.

Pour les économistes néo-libéraux, entre autres Hayek, Friedman et Buchanan, le principe d'équité est lié à la liberté fondamentale de l'individu. Ils pensent que tout impôt doit être neutre. Autrement dit, que la liberté pour l'individu de choisir sa vie (Salin, 1985, p. 215) ne doit pas être altérée par la contrainte fiscale. Je ne pense pas que cette philosophie soit plus réaliste ni plus juste que les philosophies interventionnistes qui sont à la base de l'État-providence. L'impôt n'est pas neutre et ne doit pas être neutre. Pour modeler le rôle des gouvernements, l'impôt moderne reste le meilleur outil qui soit. C'est donc sur le rôle de l'État que doit se fonder la redéfinition de l'équité.

Quelle serait donc la nouvelle norme de l'équité? Au sens strict, elle serait atteinte lorsque le fardeau fiscal serait proportionnel aux biens et services reçus. Le contribuable paierait alors un juste prix pour les produits qu'il consomme. En apparence, le plus démuni semblerait alors le plus pénalisé. Mais rien n'est moins certain. On sait que les plus favorisés, c'est-à-dire les riches contribuables et les entreprises, utilisent davantage les ressources collectives (routes, aéroports, communication et dépenses fiscales) que ne le font les citoyens dits économiquement et socialement faibles. Les budgets de dépenses sont des investissements économiques au même titre que les programmes de sécurité sociale, d'où leur grande utilité pour les leaders économiques. Dans ce cadre, l'État-arbitre, c'est-à-dire l'État protecteur des richesses et ressources collectives (dont les préoccupations d'écologie et d'environnement ne sont que les signes avant-coureurs), pourrait imposer aux 
contribuables un fardeau fiscal équivalant à la consommation (destruction) de richesse. La pénalité serait proportionnelle (voire progressive) à la consommation des richesses collectives. Dans une société, la consommation des ressources de plus en plus rares est un signe d'appauvrissement. On a l'habitude de juger les pays à la hauteur de leur niveau de vie, sans égard à la destruction massive des ressources collectives (généralement on parle de transformation des richesses naturelles). Beaucoup de ces richesses, telles que les matières premières et l'énergie, ne sont pas renouvelables. $\mathrm{Si}$ nous ajoutons que les méthodes de production modernes sont souvent responsables de la pollution, on peut dire que l'action combinée de la consommation et de la pollution entraînera la raréfaction des ressources. Tout cela mène inéluctablement à un appauvrissement. Pour être équitable, la fiscalité de l'État-arbitre devrait donc exiger des contribuables un juste prix pour la part des richesses qu'ils détruisent par la consommation (on consomme aussi bien une matière première qu'une bouteille de vin). Le principe du «pollueur-payeur», que les gouvernements instaurent en douce, est une forme d'impôt à la consommation dont l'équité est appréciable. Il faudrait étendre ce principe à toutes les activités de l'État.

On conviendra que l'État, s'il s'en tient à son rôle actuel, ne peut croître et s'étendre indéfiniment, car son mode de financement est restrictif. Il est clair que la résistance instinctive à toute contrainte empêche l'impôt de générer efficacement les revenus nécessaires au développement. Il est donc impératif pour l'État de modifier son rôle. De producteur qu'il est, il se doit de devenir régulateur, autrement dit, de jouer le rôle d'État-arbitre. Rôle qui irait de pair avec un financement moindre. Dès lors, la fiscalité pourrait devenir plus efficace.

À propos de l'équité fiscale, Bernard Vinay (1970, p. 8) écrit: "À la règle de l'égalité dans l'impôt, qui prévalait dans le cadre classique, s'est substituée la volonté d'assurer l'égalité par l'impôt». Il voulait exprimer par là la mutation de l'État: de gendarme, il est devenu providence. Cependant, les études fiscales prouvent que cette formule, aussi intéressante soit-elle, est dépourvue de réalisme. La progressivité et la redistribution sont toujours 
restées des voeux pieux. Par contre, le principe d'équité n'est pas synonyme du principe d'égalité. Le premier se vérifie dans l'analyse du fardeau fiscal que supporte réellement le contribuable, tandis que le second est une vue de l'esprit.

La première tentative sérieuse de réforme de la fiscalité dans le Canada moderne remonte au projet Benson, élaboré en 1969. À cette époque, on affirmait la nécessité d'une réforme générale en vue de la réalisation d'importants et d'utiles projets (Benson, 1969, p. 5). Deux décennies plus tard, en juin 1987, le ministre Michael Wilson déposait un projet de refonte de la structure fiscale du gouvernement fédéral. Entre les deux, se place l'essai infructueux tenté par Allan MacEachen en 1980. Il y a là une constance qui dénote à tout le moins la nécessité de procéder périodiquement à une nouvelle harmonisation de l'impôt et du rôle des gouvernements. Ce phénomène n'est pas propre au Canada; il s'observe dans la majorité des pays industrialisés (Sorman, 1984, p. IV).

C'est par son aspect global que le présent projet de réforme du ministre Wilson se distingue des précédents. Les tentatives antérieures partaient d'une préoccupation primordiale, par exemple le manque de ressources ou l'instauration d'une nouvelle philosophie politique. Le projet Wilson doit résoudre simultanément les contraintes budgétaires, l'échec de la fonction fiscale de redistribution de la richesse, la mutation du rôle de l'État et les problèmes écologiques. La résolution de cet ensemble de problèmes requiert une transformation radicale de la structure de l'impôt, qui donne peut-être lieu au passage d'un impôt sur le revenu à un impôt à la consommation. Une telle réforme ne se fera pas sans heurt, car elle risque de bousculer une croyance, bien ancrée, dans les vertus sociales de l'imposition du revenu. Qui plus est, un changement important de la structure fiscale ne sera pas sans influencer directement l'équilibre politique entre l'État et le citoyen. C'est là l'enjeu capital de toute réforme de l'impôt. 


\section{Bibliographie}

ARDANT, Gabriel, Théorie sociologique de l'impôt, 2 tomes, Paris, Sevpen, 1965.

ARDANT, Gabriel, Histoire de l'impôt, 2 tomes, Paris, Fayard, 1971.

BÉLANGER, Gérard, Croissance du secteur public et fédéralisme: perspective économique, Montréal, Agence d'Arc, 1988.

BRACHET, Bernard, «Volonté politique et rigidité fiscale», dans Beck, Bernard et Vedel Georges (Eds), Études de finances publiques, Paris, 1984.

BRENNAN, Geoffroy et BUCHANAN, James, The Power to Tax Analytical Foundations of Fiscal Constitution, Cambridge, University Press, 1980.

BROCHIER, Hubert, Finances publiques et redistribution des revenus, Paris, Armand Collin, 1950.

BUCHANAN, James, M., The Demand and Supply of Public Goods, Chicago, Rand-McNally, 1968.

BURBANK, H.H., Public Finance in Democratic Society, vol. 1, Harvard, Harvester Press, 1986.

CANADA, Réforme fiscale 1987, Ottawa, ministère des Finances, décembre 1987.

CANADA, Réforme fiscale 1987, Ottawa, ministère des Finances, juin 1987.

CANADA, Le budget 1990, Ottawa, ministère des Finances, février 1990.

CROZIER, Michel, État modeste, État moderne, Paris, Fayard, 1987.

DAFFLON, Bernard et WEBER, Luc, Le financement du secteur public, Paris, PUF, 1984.

LAFFER, Arthur, L'ellipse ou la loi des rendements fiscaux décroissants, Bruxelles, Institutum Europaeum, 1981.

LAUFENBERGER, Henry, Histoire de l'impôt, Paris, PUF, 1959.

LEFF, Mark H., The Limits of Symbolic Reform, Cambridge, University Press, 1984. 
MATOUK, Jean, Le socialisme libéral, Paris, Albin Michel, 1987.

MEHL, Lucien et BELTRAME, Pierre, Science et techniques fiscales, Paris, PUF, 1984.

MINC, Alain, La machine égalitaire, Paris, Grasset, 1987.

MUSGRAVE, Richard A. et MUSGRAVE, Peggy B., Public Finance in Theory and Practice, 4th ed., New York, McGraw-Hill, 1984.

NICKSON, Jack N. Jr, Economics and Social Choice, New York, Mc Graw-Hill, 1971.

PEACOCK, Alan T. et WISEMAN, Jack, The Growth of Public Expenditures in the United Kingdom, Princeton, National Bureau of Economic Research, 1961.

PECHMAN, Joseph A., The Rich, the Poor and the Taxes they Pay, Boulder, Westview Press, 1986.

PECHMAN, Joseph A., What Should be Taxed Income or Expenditure, Washington, The Brookings Institute, 1980.

SALLY, Pipes et WALKER, Michael, Tax Facts Six, Vancouver, Fraser Institute, 1988.

ROSANVALLON, Pierre, La crise de l'État-providence, Paris, Seuil, 1981.

SALIN, Pascal, L'arbitraire fiscal, Paris, Laffont, 1985.

SORMAN, Guy, La solution libérale, Paris, Fayard, 1984.

TAIT, Alan, The Taxation of Personal Wealth, Urtana, University of Illinois Press, 1967.

VINAY, Bernard, Fiscalité, épargne et développement, Paris, Armand Colin, 1970.

WEBER, Luc, L'État, acteur économique, Paris, Économica, 1988. 